\title{
Difusión y transferencia del CONOCimiento CIENTÍFICO Y TECNOLÓGICO A TRAVÉS DE LAS REDES DE INNOVACIÓN
}

\author{
Dairon Alberto Arboleda Quintero ${ }^{1}$ \\ Carlos Enrique Villa Arango ${ }^{2}$
}

\section{Resumen}

Considerando que el conocimiento es el elemento fundamental en la concepción moderna de competitividad e innovación y que está íntimamente relacionado con todos los actores del tejido social, en este artículo se hace una breve caracterización de los tipos de conocimiento y su relación con la empresa, el sistema educativo, las políticas gubernamentales y la sociedad en general.

En el trabajo se abordan casos paradigmáticos, que muestran la idea que la sociedad es el centro de la innovación y al mismo tiempo constituye una red que aprende colectivamente y que la atención ya no se centra en los nodos o actores aislados, sino en la interacción entre estos.

\section{Palabras clave}

Competitividad, innovación, actores, red, difusión, transferencia.

1 Ingeniero Civil de la Universidad Nacional de Colombia. Especialista en Gestión Energética Industrial en el ITM y candidato a doctor de la Universidad del País Vasco. Investigador del Grupo Da Vinci del InstituTo TECNOLÓGICO METROPOLITANO. Dirección electrónica: dairoarboleda@itm.edu.co

2 Ingeniero Industrial de la Universidad Nacional de Colombia. Especialista en Didáctica de las Ciencias con énfasis en Matemáticas y Física de la Universidad Pontificia Bolivariana de Medellín, Colombia. Investigador del Grupo Da Vinci del INSTITUTO TECNOLÓGICO METROPOLITANO. Dirección electrónica: carlosvilla@ itm.edu.co

Fecha de recepción: 12 de marzo de 2009

Fecha de aceptación: 11 de mayo de 2009 


\section{Alostract}

Considering that the knowledge is the fundamental element in the modern conception of competitiveness and innovation and that intimately is related to all the actors of the social weave, in this article becomes a brief characterization of the types of knowledge and its relation with the company, the governmental system, policies and the society educative generally.

In the work paradigmatic cases, that are approached they show the idea that the society is the center of the innovation and that at the same time constitutes a network that learns collectively and that the attention no longer concentrates in the nodes or isolated actors, but in the interaction between these.

\section{Key words}

Competitiveness, innovation, actors, social weave, network, diffusion, transference 


\section{INTRODUCCIÓN}

Diametralmente opuesta a la concepción que se tenía varias décadas atrás, donde los recursos naturales se concebían como la ventaja económica primordial de una nación en la actualidad, competencia y conocimiento se consideran los recursos críticos de los países y las empresas en el ámbito de la competitividad e innovación ${ }^{3}$. Autores notables sobre temas relacionados con la difusión y transferencia del conocimiento a través de las redes de innovación como Drucker ${ }^{4}$,Quinn ${ }^{5}$ y Toffler ${ }^{6}$, están de acuerdo en que el poder económico y de producción de una compañía moderna se basa más en sus capacidades intelectuales y de servicio, que en los activos físicos como planta, materia prima y equipo.

Términos usuales en el mundo de la tecnología como sociedad del conocimiento, economía del conocimiento y ciudades del conocimiento, son un reflejo de la trascendencia y valoración de las capacidades intelectuales en el escenario actual de la competitividad y la innovación. El conocimiento y la información son dos conceptos que se diferencian profundamente. Se puede asociar la información con un conjunto de datos organizados y analizados en un contexto específico. Por ejemplo, la información se puede procesar y almacenar en un ordenador. El conocimiento,

3 Sin desconocer que los procesos de investigación + desarrollo deben también consultar los costos ambientales en la idea del desarrollo sostenible, pues el medio ambiente, como sistema, se deteriora y pierde condiciones, por el uso indiscriminado de los recursos naturales, lo que hace que éstos adquieran una importancia especial.

4 Peter F. Drucker (1909-2005) economista y consultor (Viena, 19 de noviembre de 1909) fue uno de los mejores referentes en cuestiones relativas a la gestión corporativa y empresarial o 'management'.

5 James Brian Quinn es profesor de Gerencia, honorario, en Dartmouth College' s Amos y en la Escuela de negocios de Hannover, de New Hampshire.

6 Alvin Toffler, (nació el 3 de octubre de 1928) es escritor y futurista estadounidense doctorado en Letras, Leyes y Ciencia, conocido por sus discusiones acerca de la revolución digital, la revolución de las comunicaciones y la singularidad tecnológica.

Peter Drucker, James Brian Quinn y John Handy han avisado, cada uno en su estilo, la aparición de una nueva economía fundamentada en la información y en la creación del conocimiento. 
más que información asimilada, es de mayor significación y permite identificar, estructurar y utilizar la información para la toma de decisiones y obtención de resultados. Hall y Andriani, 2003, asocian al conocimiento factores como la intuición, la cultura organizacional, la reputación y la teoría codificada.

En la primera parte del artículo se caracteriza el conocimiento tácito $^{7}$, confrontándolo con el conocimiento explícito ${ }^{8}$. Posteriormente, se abordan casos concretos y paradigmáticos de estrategias exitosas para crear, difundir y transferir conocimiento, como son los parques científicos, clusters de empresas ${ }^{9}$, incubadoras de negocios y tecnópolis. El artículo se enfoca hacia los clusters y parques científicos ${ }^{10}$, con el propósito de resaltar la interacción entre los diversos actores públicos y privados en el contexto de la innovación: grupos de investigación, instituciones universitarias, políticas gubernamentales, capitales de riesgo, asociaciones ecológicas, cultura heredada y sociedad en general. Amitav Rath ${ }^{11}$, 1994,

7 El conocimiento tácito se adquiere por la experiencia compartida "Aprender Haciendo". No se puede articular ni codificar fácilmente

$8 \mathrm{El}$ conocimiento explícito se adquiere normalmente en la educación formal y se puede articular y codificar fácilmente

94 Complejos industriales constituidos en un medio innovador. En estas empresas existe una fuerte relación entre investigación, desarrollo y producción. Estos complejos se han generado sin una planificación deliberada. Por ejemplo, el Valle del Silicio y la ruta 128 de Boston en Estados Unidos.

10 Un Parque Científico y Tecnológico es un proyecto, generalmente asociado a un espacio físico, que mantiene relaciones formales y operativas con las universidades, centros de investigación y otras instituciones de educación superior. Está diseñado para alentar la formación y el crecimiento de empresas basadas en el conocimiento y de otras organizaciones de alto valor agregado pertenecientes al sector terciario, normalmente residentes en el propio Parque. Posee un organismo estable de gestión que impulsa la transferencia de tecnología y fomenta la innovación entre las empresas y organizaciones usuarias del Parque.

11 Rath, Amitav. University of California, Berkeley. CEO at Policy Research International Ottawa, Canadá.

Amitav Rath, director del International de la investigación de la política. Condujo un estudio para determinar hasta qué grado se podía promover la idea de los contratos de rendimiento - modelo comercial innovador creado en América del Norte - para lograr ahorros de energía en los países del Sur, con equipos de investigación en Chile, Colombia, Ghana, China, y la India... El proyecto fue 
diferencia los procesos de difusión y transferencia de conocimiento. La difusión es un proceso no intencionado que puede darse incluso por imitación y copia. La transferencia obedece a políticas claras de cooperación entre países, empresas o en el interior de una organización de una planta a otra.

Podría decirse que la difusión o transferencia tecnológica, tomadas en el sentido de propagación o divulgación de conocimientos, viene tomando auge desde la década de los sesenta y gran cantidad de autores se han ocupado desde ese entonces a este tema, entre otras cosas por los fuertes efectos que genera dicha transferencia a nivel cultural y social. Shrum ${ }^{12}$, Shenhav ${ }^{13}$, 1995, analizan profundamente el fenómeno de la transferencia de tecnología en los países menos desarrollados o también denominados del tercer mundo. Muestran por ejemplo, cómo se requiere de una fuerte infraestructura económica para absorber y habilitar el uso del conocimiento científico, el cual debe ser pertinente y aplicable en el contexto específico. Los países adinerados están en capacidad de realizar investigación básica y los países subdesarrollados quedan a merced de los desarrollos de los anteriores. Esto genera una especie de mecanismo de subyugación ideológica que cada vez acrecienta la inequidad social y la pérdida de la identidad cultural de las naciones. Hay otros casos como el de Corea del Sur, Hong Kong, Taiwán, Singapur, México y Brasil, donde la transferencia tecnológica de Europa y Norte América, no se ve como una amenaza

financiado inicialmente por el centro de investigación internacional del desarrollo (IDRC) y el instituto internacional para el desarrollo sostenible (IISD), con la ayuda adicional proporcionada por el organismo de desarrollo internacional canadiense (CIDA), el banco mundial, y el consejo de la ciencia de la Comunicación. Los resultados del estudio fueron presentados en octubre de 2000 en la conferencia nacional del rendimiento energético en Ottawa.

12 Wesley Monroe Shrum, Jr. Department of Sociology. Louisiana State University U.S.A. Ph.D. Princeton University. Sociology. 1982. M.A. Princeton University. Sociology.

13 Yehouda Shenhav es sociólogo israelí y un teórico crítico. Lo conocen por sus contribuciones en los campos de la burocracia, de la gerencia y del capitalismo, así como por su investigación sobre la pertenencia étnica de la sociedad israelí y su relación con el conflicto palestino-israelí. 
sino como una oportunidad para mejorar la capacidad industrial y las competencias de sus profesionales.

\section{División Y CLASIFICACIÓN DEL CONOCIMIENTO}

Polanyi $^{14}(1962,1966)$ afirma que el conocimiento que posee el individuo tiene un gran componente tácito. Define como conocimiento tácito, aquel que no es verbalizable, intuitivo y desarticulado. Este conocimiento se adquiere a través de la experiencia colaborativa y es difícil de articular, formalizar y comunicar. Asocia información y conocimiento explícito.

Nonaka y Takeuchi ${ }^{15}$, 1995, dividen el conocimiento de la empresa bajo dos perspectivas. Desde el punto de vista epistemológico, hablan de conocimiento explícito y tácito. En el plano ontológico ${ }^{16}$, distinguen conocimiento individual y colectivo. Sustentan que la interacción social en el contexto específico puede ayudar a convertir el conocimiento tácito en formas explícitas a través de la verbalización, codificación y aplicación tecnológica en productos y servicios. Aunque hay grandes diferencias entre el conocimiento tácito y el explícito, en la práctica no aparecen aislados e interactúan en forma dinámica para generar conocimiento nuevo e innovación.

Sercovich ${ }^{17}$, habla de la transferencia técnica como esos elementos que se requieren para la creación y funcionamiento de

14 Karl Polanyi (25 de octubre de 1886, Viena (Austria). Científico social que trabajó en el ámbito de la antropología económica y la crítica de la economía ortodoxa.

15 El modelo de Nonaka y de Takeuchi se basa en de Polanyi la distinción entre el conocimiento tácito y explícito (1983) y proporciona una comprensión de la creación y de la gestión del conocimiento de una perspectiva japonesa de la cultura del negocio.

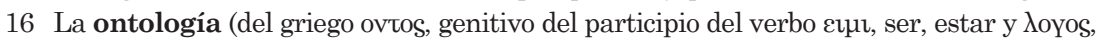
ciencia, estudio, teoría) es una disciplina que se suele identificar con la Metafísica general o bien indica una de las ramas de ésta que estudia lo que es en tanto que es y existe.

17 Francisco C. Sercovich, consultor de la Unidad de Comercio Internacional e Industria de la Sede Subregional de la CEPAL en México, en el marco del proyecto "Fortalecimiento de las capacidades relacionadas con el comercio en las Américas" (CAN/05/002). Expositor de la CEPAL en la Reunión de Expertos sobre La apertura comercial, inversión extranjera y capacidades tecnológicas. ADPIC, TLC y brechas de desarrollo. Dimensiones de política desde una perspectiva latinoamericana con el auspicio de Columbia University 
nuevas instalaciones. Algún conocimiento referente a maquinaria puede estar escrito (explícito) y otro no está codificado y reside en el personal experto (tácito).

\subsection{Conocimiento tácito}

A continuación se presentan las características básicas del conocimiento tácito que lo diferencian del explícito:

- Está empotrado en el individuo o la empresa. Habilidades, destrezas y esquemas mentales compartidos

- Se adquiere por la experiencia práctica en un contexto específico "aprender haciendo"

- Requiere de acompañamiento y contacto directo para su enseñanza y aprendizaje

- Es difícil de transmitir porque reside en el individuo y las normas sociales y culturales compartidas por años

- Es desarticulado y difícil de codificar

- Generado por ensayo y error

El proceso de transferir conocimiento tácito (tácito-tácito) es denominado socialización. El ejemplo más sencillo es el de un niño cuando aprende a hablar por imitación, con el acompañamiento de otras personas. Cavusgil, Calantone, Zhao ${ }^{18}$, 2003, sustentan que un alto grado de conocimiento tácito puede ser adquirido por la interacción cercana con empresas similares. De esta forma las empresas pueden beneficiarse de la experiencia colaborativa en los procesos de transferencia de conocimiento tácito.

Las redes de cooperación para transferir conocimiento tácito, pueden también extenderse por fuera de las fronteras regionales y nacionales. Cheng, Choi, 2004, analizan profundamente el caso del parque científico de Hsinchu en Taiwán. Los más aventajados

18 Title: Tacit knowledge transfer and firm innovation capability. Author(s): S. Tamer Cavusgil, Roger J. Calantone, Yushan Zhao. Journal: Journal of Business \& Industrial Marketing. Year: 2003 . Volume: 18 . Issue: 1 Page: 6 - 21. ISSN: 0885-8624 DOI: 10.1108/08858620310458615 Publisher: MCB UP Ltd. 
estudiantes taiwaneses viajan a cualificarse a Estados Unidos y luego regresan para aportar en la creación y modernización de capacidades locales. En uno de sus textos, Ikujiro Nonaka relata un interesante caso donde se refleja la importancia del conocimiento tácito. En la ciudad japonesa de Osaka, la compañía Matsushita Electric estaba interesada en diseñar y comercializar una panificadora de uso doméstico. Después de mucho tiempo de trabajo y constantes intentos no lograban un óptimo amasado del pan que se quemaba por fuera y por dentro quedaba crudo. Como en el Osaka Internacional Hotel se producía un pan de reconocida calidad, se optó por enviar a un investigador de la empresa para que aprendiera del panadero del Hotel.

Después de varios meses de trabajar con el panadero experto y de observar, imitar y probar iterativamente por ensayo y error; el investigador encontró la solución al problema. Incluyendo unas nervaduras especiales en el interior de la máquina, se logró un amasado similar al del hotel, que se reflejó en un sorprendente aumento en las ventas del electrodoméstico.

Este ejemplo muestra como a través de un conocimiento tácito carente de teorías y especificaciones, una empresa puede ser competitiva e innovadora. Además se observa claramente el tránsito del conocimiento tácito a explícito, cuando es compartido por el grupo de investigación.

\subsection{Conocimiento explícito}

El conocimiento explícito es privilegiado en países como Estados Unidos y el Reino Unido, ya que ha sido fundamental en el desarrollo científico y tecnológico de la humanidad. En contraste con el conocimiento tácito, el conocimiento explícito presenta las siguientes características:

- Es objetivo y aceptado universalmente

- Se aprende en la educación formal sin necesidad de un contexto específico

- Se puede adquirir a distancia sin acompañamiento directo 
- Fácil de comunicar y transmitir

- Es articulado y fácil de codificar

- Generado por deducción lógica

El proceso de comunicar conocimiento explícito (explícito a explícito) se denomina combinación o diseminación. El conocimiento colectivo-explícito se utiliza en señales y símbolos, códigos, recetas, reglas y procedimientos en una empresa. Es público y puede usarse en ocasiones sin grandes requerimientos intelectuales; como por ejemplo, llevar la contabilidad de una firma en una hoja de cálculo. Por ser mecánico y enciclopédico el conocimiento se vuelve un programa estandarizado.

La conversión de conocimiento explícito a tácito se denomina interiorización o internalizacion. Es el caso de dos músicos que disponen de los mismos códigos para interpretar una pieza musical, pero que por la experiencia o la práctica, uno la ejecuta mejor que el otro.

A partir de casos reales, autores como Nonaka y Takeuchi, han encontrado fuerte correlación entre el tipo de organización y la interacción entre conocimiento tácito y explícito. En las empresas con estructura jerárquica (vertical) se favorecen los procesos de combinación e interiorización, mientras las organizaciones con estrategia horizontal son más adecuadas para la socialización y la exteriorización.

En la transferencia de conocimiento científico y/o tecnológico entre empresas o países, de los cuales unos pueden ser explícitos y otros tácitos, (Amitav Rath, 1994) enuncia once mecanismos básicos:

1. Libros, estándares, información de patentes

2. Educación y entrenamiento en el exterior

3. Reuniones, conferencias y visitas a los sitios de interés

4. Intercambio de información y personal con programas técnicos de cooperación

5. Empleo de expertos y consultores extranjeros 
6. importación de maquinaria y equipos con su información técnica

7. Ingeniería inversa ${ }^{19}$

8. Especificaciones técnicas, estándares y entrenamiento proporcionado por los importadores

9. Acuerdos para utilizar patentes y conocimientos técnicos propios

10. Inversión directa extranjera que trae todos los elementos de conocimiento técnico necesarios

11. Importación de productos de tecnología intensiva

\section{El CONOCIMIENTO Y SU RELACIÓN CON ACTORES}

\section{SIGNIFICATIVOS EN EL ESCENARIO DE LA INNOVACIÓN}

Como se había expresado anteriormente, en la concepción moderna de innovación ${ }^{20}$ la atención no se centra en los nodos o actores sino en las relaciones entre estos. Ya no es suficiente el modelo de triple hélice que se enfoca en la interacción universidadindustria-Estado, porque entran en escena otros actores como los grupos ecologistas o defensores del medio ambiente, que luchan por racionalizar el avance tecnológico fijándole límites y restricciones; la iglesia que se aferra a los principios naturales en que se desarrolla la sociedad y la inversión de la banca privada en empresas de alta tecnología. Proyectos tan ambiciosos como los reactores de fisión nuclear para generar energía, los satélites artificiales y los viajes tripulados al espacio, no serían una realidad, si naciones enteras no se lo hubieran impuesto como una meta.

19 Ingeniería inversa: es revertir el proceso de diseño de tecnología en el sentido de invertir los pasos de i + D. es como tomar un equipo que está en el mercado y desagregarlo en sus sistemas constitutivos básicos, para luego mejorarlo o copiarlo. Los japoneses usaron esta práctica en la década de los 60 a 70.

20 La innovación no es un accidente, sino un proceso. Es algo que se administra, se mejora a lo largo del tiempo y se utiliza para lograr metas explícitas de crecimiento corporativo. Esta es la concepción moderna de la innovación, la que aplican las empresas de primer orden en el mundo, desde Microsoft hasta Procter \& Gamble y BMW. 
En este artículo no se enfatiza en actores como la iglesia o los grupos ecologistas, no por considerarlos como un obstáculo para la innovación y difusión del conocimiento científico, sino porque se quiere resaltar el papel en las relaciones de unos actores específicos: las instituciones de educación superior, la empresa, el estado y el entorno cultural y social

Basándose en casos concretos estudiados en países y empresas específicas, Nonaka, Tekeuchi y otros autores como Alice Lam ${ }^{21}$ (1997, 2000), han encontrado una alta correlación entre el conocimiento generado y las estructuras educativas, laborales y sociales. Alice Lam hace una importante caracterización de cuatro tipos de organizaciones:

- Burocracia profesional. Son empresas fundamentadas en el conocimiento individual y estandarizado. El conocimiento tácito no es valorado y son empresas poco innovadoras.

- Burocracia formal. Estas organizaciones están basadas en el conocimiento colectivo estandarizado. Hay un distanciamiento apreciable entre los altos directivos y el trabajador de planta. No son empresas preparadas para la innovación, porque se limitan a resolver problemas rutinarios, lo que hace que se subvalore el conocimiento tácito y la mentalidad creadora de los individuos.

Sin desconocer la importancia de las normas internacionales de calidad (ISO 9000, MIL, NEMA, y otras), no se puede pretender una estandarización rígida de todos los procesos técnicos y administrativos de la empresa. Hay que reservar un espacio para el ingenio y la creatividad colectiva, como factor decisivo para la generación y diseminación del conocimiento tácito.

- La adhoc-cracia activa. Son organizaciones fundamentadas en el conocimiento individual no estandarizado. Se valora significativamente tanto el conocimiento formal que poseen

21 Profesora adjunta de Recursos humanos y conducta organizativa, en la Universidad de Kent. Sus investigaciones se centran en la administración comparada, el aprendizaje de la organización y la innovación, y la organización empresarial japonesa. 
sus empleados, como las competencias que adquieren los expertos individuales para resolver problemas prácticos. Son el escenario ideal para generar conocimiento tácito a través de la experimentación y solución de problemas nuevos. Las empresas de diseño de software son el ejemplo típico de este modelo de organización.

- La organización adaptada al trabajo. Estas empresas tienen como base el conocimiento colectivo no estandarizado. El conocimiento está integrado en las rutinas, el trabajo en equipo y la cultura común. Son empresas innovadoras preparadas para el cambio, donde se crea, distribuye y acumula el conocimiento tácito a través de la experiencia compartida (aprender haciendo).

En la multinacional ensambladora de automóviles Sofasa-Renault, los operarios de planta han propuesto importantes innovaciones, incentivados por bonificaciones económicas o por el mero hecho de sentirse partícipes del proceso. Otro caso interesante tiene que ver con la empresa Colgate-Palmolive, que cambió el inadecuado sistema de tapa rosca de las cremas dentales, por iniciativa de la señora que reparte café en las oficinas.

El sistema educativo predominante en un país, es determinante en el tipo de conocimiento generado y distribuido:

- En el sistema de educación elitista cerrado se otorga mucha importancia al conocimiento formal frente a las competencias y habilidades de tipo práctico. Este es el caso de Estados Unidos y el Reino Unido, cuyo sistema educativo conduce a estructuras empresariales jerárquicas, donde hay una gran distancia social entre los directivos y empleados de planta.

Hay un caso que llama la atención en la ciudad de Medellín (Colombia), de una familia que se ha dedicado por más de cincuenta años a la reparación exclusiva de cámaras fotográficas de todo tipo de especificaciones. Es tal la competencia que han adquirido los miembros de esta familia, que uno de sus principales clientes fue el famoso periódico The New York Times. Parece inexplicable que desde un país tan desarrollado 
tecnológicamente, se busque la solución a problemas técnicos en un país subdesarrollado como Colombia. Este hecho confirma, que en los países del norte hay una carencia de personas con cualificación intermedia entre los muy especializados y los pocos idóneos en conocimientos específicos ${ }^{22}$. Su sistema educativo cerrado y elitista, no favorece la adquisición de conocimiento tácito y competencias práctico-experimentales.

- En el sistema abierto igualitario de educación, se da importancia tanto a la educación académica formal como a la formación profesional en competencias, lo que favorece la difusión del conocimiento tácito. Es el caso de Dinamarca, Japón y Alemania, donde el sistema educativo conduce a estructuras empresariales descentralizadas

Es muy importante que se vaya observando la gran correlación entre los distintos elementos y factores que intervienen en el contexto de la innovación.

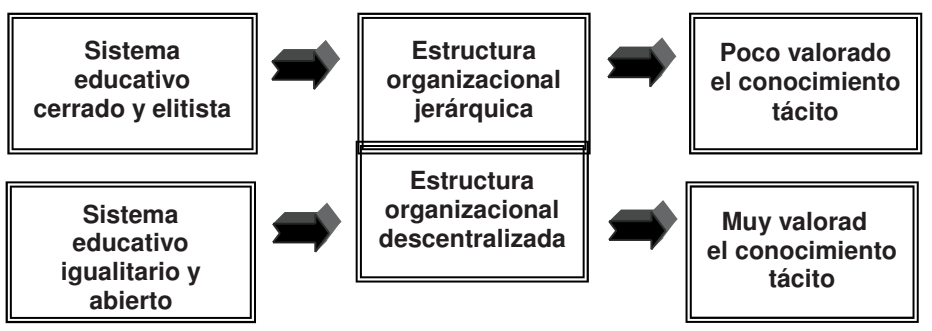

Alguien se podrá preguntar: ¿Por qué si el conocimiento tácito y los sistemas educativos abiertos son tan decisivos en la innovación; Estados Unidos sigue siendo el país más poderoso del mundo?

Así como los efectos positivos de las redes de innovación se perciben después de largos períodos de tiempo, un fenómeno similar ocurre con los efectos negativos. Hay que observar es cómo están ganando el mercado otros países y qué podrá ocurrir después de veinte años o más.

22 Laicismo y sistemas educativos en Europa: ¿una Europa laica? Por Antonio Gómez Movellán 
El mercado laboral es otro factor que puede influir en la creación y difusión de un conocimiento específico; pero parece no ser tan determinante respecto al conocimiento tácito, como sí lo son el sistema educativo y las estructuras organizacionales. Por ejemplo, en el Valle del Silicio se combina un mercado laboral amplio con posibilidades de rotación de una empresa a otra, con un poderoso conjunto de redes profesionales y sociales, generando la concentración de empresas más innovadoras y competitivas del mundo. En el Japón fundamentan la capacidad de innovación incremental ${ }^{23}$ en la acumulación de saberes tácitos y competencias prácticas de sus empleados de planta, combinados con un mercado laboral interno basado en las empresas, donde la persona goza de estabilidad y asciende en la estructura jerárquica en la medida en que se especializa en el campo de acción de la compañía.

En un mercado laboral amplio, la difusión del conocimiento tácito requiere de una estructura social que lo contenga, dado que los individuos son muy autónomos y se necesita transferir sus conocimientos a las empresas para estimular la innovación radical $^{24}$. En este aspecto toman gran importancia las redes de empresas, los parques tecnológicos y los clusters o concentraciones industriales localizadas en un entorno apropiado.

\subsection{El papel de la universidad en la creación y transferencia de conocimiento}

En el momento de analizar el papel que juega la universidad en la generación y difusión de conocimiento científico o tecnológico, no se debe generalizar porque se refiere a contextos muy específicos con un entorno social, cultural y económico muy diferente. Por ejemplo, las universidades de Stanford y Cambridge han jugado un papel decisivo en la generación de conglomerados de empresas

23 Cuando se mejora un proceso o un producto que ya existe en el mercado o las empresas. Por ejemplo, disminuir el tamaño de los ordenadores con las mismas o mejores posibilidades de cálculo.

24 Es una innovación que causa enorme impacto y cambia parcial o completamente el paradigma dominante. Por ejemplo el paso del tubo de vacío al transistor. 
de base tecnológica, como resultado de un gran prestigio en la investigación básica y aplicada y los vínculos fuertes alcanzados con el sector industrial. No todas las universidades tienen estas potencialidades para ser los actores principales en el escenario de la innovación y se debe ser muy consciente de estas limitaciones. Es el caso de muchas universidades latinoamericanas, que pretenden convertirse de un día para otro en centros de transferencia tecnológica. Más bien, se deberían centrar en el estudio serio del mundo de la innovación y las distintas relaciones entre los diversos actores en este contexto.

En un país industrializado o en vías de industrialización, la universidad puede intervenir activamente en tres aspectos:

- Generar nuevo conocimiento científico básico y aplicado a través de sus grupos de investigación, con el apoyo del sector público y privado.

- Formación con alta calidad de científicos, ingenieros, técnicos, administradores y demás profesionales, que satisfagan las demandas del comercio y la industria.

- Cristalizar los avances en la investigación, creando una red de empresas industriales y de nuevos negocios.

Hay otros aspectos de orden legislativo, donde la universidad debe procurar un ambiente propicio para atraer y retener a los académicos de prestigio. Por ejemplo, que no se ofrezca un sueldo cerrado a los catedráticos y que no se les exija exclusividad con la universidad para que puedan generar sus propias empresas.

\subsection{El papel del estado}

En mayor o menor escala, siempre el Estado es un actor de primera línea para alcanzar el desarrollo comercial e industrial de un país. La intervención del Estado no radica solo en su capacidad para fomentar I + D + i (Investigación, Desarrollo, innovación), sino en procurar un entorno favorable al desarrollo, flexibilizando las regulaciones y requerimientos legales y estructurando un sistema educativo acorde a las demandas de la sociedad. 
La intervención del Estado ha sido muy decisiva en países como Japón, Taiwán y Corea del Sur, donde el gobierno procura el bienestar socio-económico impulsando empresas competitivas e innovadoras.

La implementación de políticas públicas en ciencia y tecnología, están asociadas con grandes efectos sociales y económicos, como muestran los siguientes ejemplos:

- En Estados Unidos en 1980 se creo una Ley que permite patentar descubrimientos con dineros públicos. Esto trajo como consecuencia un gran auge de la industria biomédica, que generó 200.000 puestos de trabajo ${ }^{25}$.

En los países latinoamericanos y específicamente en Colombia, aun hay que lograr consenso dentro de los políticos con respecto a la aprobación de una ley de éstas.

- Con el apoyo de Estados Unidos a mediados del siglo XX, el Gobierno Taiwanés le compra las tierras a los grandes terratenientes, con los objetivos de que estos fundaran industrias y alquilar la tierra a los campesinos para que produjeran alimentos. El ministerio impulsó la industria presada, la producción de electricidad, la telefonía y la construcción de carreteras. Para 1965 Taiwán ya no dependía económicamente de Estados Unidos y se crea la primera zona industrial exenta de impuestos internos, lo que favoreció la fundación de poderosas petroquímicas y metalúrgicas.

En 1980, Taiwán entra en la alta tecnología, creando el parque científico-tecnológico en Hisinchu con más de cien empresas que generan 25.000 empleos. Para preservar el medio ambiente, se construye en Taipei una planta de tratamiento con capacidad de 30.000 toneladas diarias, en un paisaje donde abundan los pájaros, arroyos y vegetación fresca.

No todos los países tienen las mismas posibilidades de combinar una ciudad industrializada con un ambiente sano y limpio. Taiwán

25 Joan Josep Guinovart: "Barcelona será el gran centro de biomedicina del Sur" Entrevista: JOAQUIM ELCACHO. Revista Barcelona Metrópolis Medeteránea, No. 55 
sólo produce el 15\% de los alimentos que se consumen internamente, lo que disminuye el uso de pesticidas y otros productos que alteran el ecosistema.

\subsection{La cultura y la organicación social en el contexto de la innovación}

La innovación tecnológica no es el resultado de combinar deliberadamente conocimiento científico, personal calificado, capital de riesgo, políticas institucionales y muchos otros factores. Se requiere primordialmente de un sistema socio-cultural preparado y comprometido colectivamente con estos ambiciosos proyectos.

Por ejemplo, que en un día lluvioso, una persona encuentre y disponga de un paraguas en el metro de Tokio para luego voluntariamente devolverlo a su sitio es algo impensable en los países latinoamericanos. El respeto por el otro, el sentido de cooperación mutua, la honestidad de los políticos y el espíritu emprendedor; son rasgos fundamentales para crear una red social de innovación fuerte y preparada para competir. Se ha probado con casos reales, como en culturas diferentes con similares condiciones económicas y de infraestructura, no se obtienen los mismos resultados en proyectos de innovación.

Hay dos actores que son muy afectados por el contexto social y cultural en el que intervienen: la religión y los grupos defensores del medio ambiente.

Según Chingiz Aitmatov, diplomático y escritor, embajador kirguiz ante la OTAN, la Unión Europea y el reino de Bélgica: "El fundamentalismo y el extremismo religiosos surgidos en Afganistán constituyen una manifestación de barbarie, un retroceso al feudalismo medieval. En otras palabras, lo que hemos contemplado era un choque de civilizaciones inevitable."

El tema de la defensa del medio ambiente debe manejarse a nivel macro y con intervención directa de organismos como la ONU (Organización de la Naciones Unidas), porque abordado a nivel local, se está convirtiendo en un gran obstáculo para el desarrollo de las ciudades. "Se exige seguridad, pero sin policía". 
Es difícil encontrar localidades que acepten la construcción de un relleno sanitario. Al generar energía diseñando grandes represas, hay que planear el traslado de los moradores hacia otro entorno. En lo que respecta a la energía nuclear, es mucho lo que hay que prepararse para desarrollarla cumpliendo con los parámetros legales en lo concerniente con el cuidado del medio ambiente. Por todos estos aspectos, ahora más que nunca, se necesita que la sociedad se convierta en una verdadera red, donde múltiples actores interactúan para optimizar los recursos.

Las redes sociales de información, que varían de acuerdo al entorno cultural, acompañadas de una cultura empresarial dispuesta al progreso, son elementos esenciales para optimizar el entorno de innovación. Estas redes no se forman en un lapso de tiempo estipulado como resultado de políticas generales. Tomando la experiencia de algunos países, se ha observado que se requieren entre diez y treinta años para cuantificar el verdadero impacto de un programa ambicioso de innovación ${ }^{26}$, siendo el entorno social un factor decisivo.

\section{Casos significativos en la creación de un entorno PROPICIO PARA La INNOVACIÓN}

Ya se había mencionado que los clusters de empresas y los parques tecnológicos ${ }^{27}$, son ambientes propicios para la generación

26 Por ejemplo, como indica Drucker (1998: 156): 'Las innovaciones centradas en el conocimiento tienen el tiempo de desarrollo (lead time) más largo de todas las innovaciones... Visto globalmente, el tiempo de desarrollo es algo así como de 50 años, una cifra que no se ha reducido de manera apreciable a lo largo de la historia.' De modo similar, Georghiou (1998) señala que puede transcurrir un tiempo considerable hasta que los efectos de un proyecto se hagan evidentes, por ejemplo, refiriéndose a un estudio en Noruega que concluyó que se requerían unos 12 a 15 años para que los resultados se hicieran palpables. Como observa el autor, este hecho tiene implicancias significativas para la forma de hacer el seguimiento de la parte práctica

27 Para Paloma García, gerente del Parque Científico de Madrid, la diferencia entre un parque científico y uno tecnológico es que en el primero debe haber al menos una universidad involucrada. En el parque de Madrid han abierto dos incubadoras de empresas. "En la primera, a los cuatro meses de ponerla en marcha ya había 42 licenciados y doctores trabajando". 
y distribución del conocimiento. Los parques científicos son el resultado de políticas intencionales direccionadas a optimizar las interacciones entre los distintos agentes que intervienen en el proyecto. Los clusters en contraste, se generan espontáneamente en un ambiente propicio para su desarrollo. A continuación se presentan dos casos exitosos relacionados con estos conglomerados de empresas innovadoras y donde interactúan universidad, empresa, estado y sociedad en general.

\subsection{El valle del silicio}

Este gran complejo tecnológico está ubicado en California, en la costa Oeste de los Estados Unidos. Es el primer núcleo industrial que hacia 1949, impulsado por la universidad de Stanford, establece en la práctica la relación entre ciencia y desarrollo económico. Allí se establecieron empresas especializadas en microelectrónica e informática. Innovaciones como el circuito integrado, el sistema Unix y el ordenador personal, resultaron de la investigación compartida en este contexto.

El Pentágono y la Nasa fueron grandes financistas para apoyar las actividades de I + D de muchas empresas de microelectrónica de la región. De siete empresas en 1955 se pasó a noventa en 1980 y en la actualidad se genera empleo para más de 300.000 personas con alta cualificación académica. En el Valle del Silicio, el conocimiento se difunde y transfiere a través de redes informales (persona a persona) con la significativa participación del capital de riesgo y organizaciones profesionales, que han posibilitado innovaciones radicales como el ordenador personal.

\subsection{El parque científico-tecnológico de Hisinchu, en Taiwan}

A diferencia de otros centros tecnológicos como el de Cambridge en el Reino Unido, que históricamente han tenido condiciones favorables para la innovación; este parque se creo en 1980 en una de las zonas menos industrializadas de la isla de Taiwán. El fuerte apoyo económico del Gobierno Taiwanés ha sido el factor primordial para la cristalización del parque, sumado a los aportes de las 
universidades nacionales de Chiaotung y Tsinghua ubicadas en la zona y al Instituto de Investigación de Tecnología Industrial, que cuenta con cinco organizaciones dedicadas a la investigación.

Son de resaltar las políticas públicas tendientes a atraer empresas nacionales y extranjeras al parque, relacionadas con exenciones fiscales, capitalización de las patentes y los programas de capacitación para ingenieros que trabajen en la zona.

En 1980, Taiwán fue el país que envío más alumnos a doctorarse en Estados Unidos, que luego repatrió para explotar el conocimiento adquirido por éstos en el Valle del Silicio. En el 2001 se disponía de 2.991 patentes de 335 compañías y las tres empresas taiwanesas productoras de circuitos integrados desarrollan sus actividades en el parque. Estas son el resultado de la inversión privada sumada al capital público.

El éxito del parque se ha basado en tres actividades básicamente:

- Creación de conocimiento local

- Transferencia de conocimiento tácito del Valle del Silicio en California

- Transformación del conocimiento en actividades productivas para crear un sistema de aprendizaje continúo

\section{Conclusiones}

El objetivo fundamental de este artículo, es mostrar como el conocimiento es un elemento transversal en las redes de innovación, ya que se relaciona directa o indirectamente con todos los actores en este contexto (los afecta y puede ser afectado por estos), tales como el sistema educativo, el tipo de organización o las políticas estatales sobre ciencia y tecnología. También se pretende reforzar la tesis, de que la innovación nace primordialmente de una red centrada en la sociedad donde ningún agente puede actuar en forma aislada. De acuerdo a esto, se pueden resaltar unos puntos básicos:

- Las estructuras organizacionales jerárquicas donde hay una gran distancia entre los directivos y los empleados de planta, no 
favorecen la generación y distribución de conocimiento tácito, el cual está más asociado con estructuras organizacionales descentralizadas donde el operario está en contacto permanente con los administradores y gerentes, posibilitando su participación activa en los procesos de innovación.

- El sistema educativo de un país, es un factor determinante en la caracterización de las estructuras organizacionales (jerárquicas, descentralizadas) y por tanto en el tipo de conocimiento privilegiado (tácito, explícito). Mientras haya una buena remuneración e incentivos para los empleados, el mercado laboral no es un factor determinante en la generación y transmisión de conocimiento. Tanto el mercado laboral amplio y abierto como el empleo estable por muchos años en una empresa, posibilitan la adquisición de competencias de los empleados que se traduce en productos de innovación.

- Muchas empresas tratan de eliminar sistemáticamente el conocimiento tácito, por temor a que sus empleados al dejar la compañía sean difíciles de reemplazar o se vayan con este conocimiento. Por el contrario, hay empresas que encuentran en la acumulación de conocimiento tácito la gran ventaja competitiva. Lo ideal es que se estimule la combinación adecuada del conocimiento tácito con el explícito, y así las empresas estarán preparadas para la innovación y transferencia de conocimiento en un entorno volátil e inestable.

- Las redes sociales de información interactuando con fuertes asociaciones profesionales, en un marco propicio para la innovación como son los clusters de empresas o ciudades basadas en el conocimiento, han sido la clave de experiencias exitosas como el Valle del Silicio y el parque científico en Hsinchu Taiwán.

- En los países en vía del desarrollo se deben estudiar profundamente estas experiencias, no con el propósito de igualarlas o superarlas, sino para fijar políticas tendientes a mejorar el panorama de la innovación a largo plazo. 


\section{Referencias BIBLIOGRáficas}

AGUIRRE, Carlos y REBOIS, Rolan. Ciencia, tecnología e innovación: Conceptos y prácticas. Universidad Andina. Proyecto de Monitoreo de Nuevas Tecnologías. Sucre (Bolivia), 1994.

ÁLVAREZ HEREDIA, Benjamín y GÓMEZ BUENDÍA, Hernando. Ciencia y tecnología. Retos del Nuevo Orden Mundial para la capacidad de investigación en América Latina. CIID, Instituto de Estudios Liberales Tercer Mundo Editores, Bogotá, 1993.

BAGLIETTO, Alicia. BALLESTEROS, Alberto. HACIA UNA ECONOMÍA DEL CONOCIMIENTO. Editorial: ESIC. 2001. Páginas: 203

CAÑAS, Raúl; LAVADOS, Jaime y MARCOVITCH, Jacques. Gestión tecnológica y desarrollo. Serie Manuales I\&D. Cinda/Pund/Secab. Santiago de Chile, 1989.

CASTELLS, Peré y VALLS P, Jaume. Tecnología e innovación en la empresa. Dirección y gestión. Barcelona, Edicions Universitat Politécnica de Catalunya. 1997.

CHAPARRO, Fernando. Conocimiento, innovación y construcción de sociedad: Una agenda para la Colombia del Siglo XXI. Santafé de Bogotá, 14 de agosto de 1998.

COLCIENCIAS. Desafíos en la construcción de una sociedad del conocimiento. Informe de cuatrienio 1995 - 1998. Santafé de Bogotá, Julio de 1998.

COLCIENCIAS. Sistema Nacional de Innovación. Nuevos escenarios de la competitividad; Ciencia y Sociedad: Colombia frente al Reto del Tercer Milenio. Santafé de Bogotá, septiembre de 1998.

COLOMBIA: Ciencia \& Tecnología. La Innovación Tecnológica como Factor de Desarrollo. Trece casos exitosos. Vol. $15 \mathrm{~N}^{\circ}$ 4, octubre- diciembre de 1997.

COLOMBIA. Al filo de la oportunidad. Misión de Ciencia, Educación y Desarrollo. Presidencia de la República. Consejería para la Modernización del Estado- Colciencias. 21 julio de 1994.

CAVUSGIL, S. Tamer; CALANTONE, Roger J. and ZHAO Yushan. Tacit knowledge transfer and firm innovation capability. The journal of business \& Industrial Marketing: 2003; 18, 1. 
CHEN, Stephen and CHOI, CHONG JU. Creating a knowledge-bases city: the example of Hsinchu Science Park. Journal of Knowledge management. Vol 8 No. 5, 2004, pp. 73-82.

DRUCKER, Peter F. La innovación y el empresario innovador. Editorial Sudamericana. Páginas: 320 . 1994

DRUCKER, Peter. "The Discipline of Innovation", Harvard Business Review, May-June 1985

DRUCKER, Peter F. Una nueva dimensión de la administración. Edit. Norma, Colombia. 1987.

DRUCKER, Peter F. La gerencia. Edit. Ateneo, Argentina. 1997.

ESCARDINO BENLLOCH, Agustín. Reflexiones sobre la I+D, la universidad y las empresas Industriales. Universidad Jaime I. Castelló de la Plana, septiembre 27 de 1995.

HALL, Richard and ANDRIANI, Pierpaolo. Managing knowledge associated with innovation. Journal of business research 56 (2003) $145-152$.

JARAMILLO, Luis Javier. La otra cara empresarial de Colombia. Qué hacen y cómo las empresas innovadoras en Colombia. Tercer Mundo/Tecnos. 1997.

JARAMILLO, Luis Javier. "Difusión de Tecnología en el Japón”. Ciencia, Tecnología y Desarrollo. Bogotá, 2, (1): 1- 146, enero - marzo, 1978.

JARAMILLO, Luis Javier. "La cooperación y la integración latinoamericana en ciencia y tecnología. ¿Balcanización o comunidad de esfuerzos?” En la cooperación internacional y el desarrollo científico y tecnológico. Balance y perspectivas. BID-CINDA-SECAB. Santiago de Chile, diciembre de 1991.

LAM, Alice. Los modelos societales alternativos de aprendizaje e innovación en la economía del conocimiento. En : http://www.campus-oei.org/salactsi/lam.pdfhttp:// www.campus-oei.org/salactsi/lam.pdf. (25, mayo, 2005).

LAM, Alice. Tacit knowledge, organizational learning and innovation: a societal perspective. Octubre 1988.

NONAKA, I., TAKEUCHI, H. (1995). The Knowledge-Creating Company. USA: Oxford University Press.

MAYORGA, Román. Sí se puede. Casos de innovación tecnológica en América Latina. Primer Borrador. BID/SOC/SDS. Washington, D.C. Mayo de 1997. 
MINTZBERG, Henry; QUINN, JamesB.; VOYER, John. El Proceso estratégico : conceptos, contextos y casos : edición breve (1997)

OSPINA T., Alberto "La vacuna de Patarroyo contra la Malaria. Trayectoria de una idea”. Innovación y ciencia. Volumen III, Nº 1.1994.

POLANYI, Karl. The Great Transformation. Traducción española: La gran transformación, Madrid, La Piqueta, 1989.

POLANYI, Karl. ARENSBERG, Conrad M., PEARSON, Harry W. Trade and Markets in the Early Empires (1957) -Comercio y mercado en los imperios antiguos[4], Barcelona, Labor, 1976.

POLANYI, Karl, The Livelihood of Man, edición de H.W. Pearson, 1977 trad. esp. El sustento del hombre, Barcelona, Mondadori, 1994.

QUINN, James Brian. Strategy, Science and Management (2002). Prentice Hall

QUINN, James Brian. Strategic Outsourcing: Leveraging Knowledge Capabilities. Sloan management review. 1999

RATH, Amitav. Notes from the Field. Comparative Technology Transfer and Society - Volume 4, Number 1, April 2006.

ROCA, William M. "El papel de la biotecnología en la agricultura de los países en desarrollo". Innovación y Ciencia. Volumen II, $\mathrm{N}^{\circ} 1$, eneromarzo de 1993.

SÁBATO, Jorge A. "Bases para un Régimen de Tecnología”. En Karl-Heinz Stanzick y Peter Schenkel (editores) Ensayos sobre política tecnológica en América Latina. Instituto Latinoamericano de Investigaciones Sociales. Quito, 1974.

SENGE, Peter. Diagnosing and Changing Organizational Culture; Addison Wesley, USA, 1999

SHRUM, W, Y. Shenhav. "Science and Technology in Less Developed Countries", en Sheila Jasanoff, G. Markle, J. Petersen. T. Picnh (eds.), Handbook of Science and Technology Studies, Londres. SAGE, 1995, 627651.

SHRUM, W. Information Society and Development: The Kerala Experience. Edited by Antony Palackal \& Wesley. 2007. Rawat Books

SERCOVICH, Francisco C. Negociación y explotación de tecnología licenciada desde el exterior: el caso de las industrias química y petroquímica (Colección Política Científica y Tecnológica) . 1975. OEA. Buenos Aires 
SERCOVICH, Francisco C. Tratados de libre comercio, derechos de propiedad intelectual y brecha de desarrollo: dimensiones de política desde una perspectiva latinoamericana. Unidad de Comercio Internacional e Industria. México, D. F., junio de 2008. estudios y perspectivas. S E R I E SEDE SUBREGIONAL D E L A C E P A L EN MÉXICO

SERCOVICH, Francisco C. Financiamiento de la innovación y cambio tecnológico., Presentado en: Seminario Internacional de Financiamiento a la innovación tecnológica, Caracas, 1986.

SERCOVICH F.C. y DAHLMAN, C.J. Local Development and Exports of Technology. The Comparative Advantage of Argentina, Brazil, India, the Republic of Korea, and Mexico.

SERCOVICH, Francisco C y TEUBAL, M. (2007), "Innovation, technological competence and competiveness: the policy issues in evolutionary perspectiva", Nueva York, inédito.

TOFFLER, Alvin. La Tercera Ola. Editorial Plaza \& Janes Editores S.A. 1979. Páginas: 525

TOFFLER, Alvin. El cambio de poder. Editorial Plaza \& Janes Editores S.A. 1992. Páginas: 525

TOFFLER, A. El cambio del poder. Ed. Plaza \& Janes, Barcelona, 1990.

Schumpeter Joseph A, .Teoría del desenvolvimiento económico. Editorial. Fondo de Cultura Económica. Editorial: Fondo Cultura (Mexico). 1997. Páginas: 254

TOFFLER, Alvin. El shock del futuro. 1970. Editorial Plaza \& Janes Editores S.A. Barcelona. 1990.

VAN DE VEN, Andrew H. The context-specific nature of competence and corporate development. Asia Pacific journal of management, 21, 123 147, 2004.

UNCSTD-UNCTAD-COLCIENCIAS. Productividad, innovación y desarrollo tecnológico en Colombia: situación actual y políticas de fomento. Informe Preparatorio para la Misión de Evaluación. Ejercicio de evaluación de la política de innovación tecnológica y de desarrollo científico en Colombia. Santafé de Bogotá, Junio de 1996.

YOUNG, Norton. Desarrollo de tecnologías autóctonas. En Seminario sobre Transferencia e Innovación de Ciencia y Tecnología. Instituto de Tecnología. Instituto de Integración Cultural. Editorial Bedout. Medellín, 1972. 